\title{
Repetitive Intermittent Hypoxia Induces Respiratory and Somatic Motor Recovery after Chronic Cervical Spinal Injury
}

\author{
Mary R. Lovett-Barr, ${ }^{1 \star}$ Irawan Satriotomo, ${ }^{1 \star}$ Gillian D. Muir, ${ }^{2 \star}$ Julia E. R. Wilkerson, ${ }^{1}$ Michael S. Hoffman, ${ }^{1}$ \\ Stéphane Vinit, ${ }^{1}$ and Gordon S. Mitchell ${ }^{1}$ \\ ${ }^{1}$ Department of Comparative Biosciences, University of Wisconsin, Madison, Wisconsin, 53706, and ${ }^{2}$ Department of Biomedical Sciences, Western College \\ of Veterinary Medicine, University of Saskatchewan, Saskatoon, Saskatchewan, Canada, S7N 5B4
}

\begin{abstract}
Spinal injury disrupts connections between the brain and spinal cord, causing life-long paralysis. Most spinal injuries are incomplete, leaving spared neural pathways to motor neurons that initiate and coordinate movement. One therapeutic strategy to induce functional motor recovery is to harness plasticity in these spared neural pathways. Chronic intermittent hypoxia (CIH) (72 episodes per night, 7 nights) increases synaptic strength in crossed spinal synaptic pathways to phrenic motoneurons below a C2 spinal hemisection. However, $\mathrm{CIH}$ also causes morbidity (e.g., high blood pressure, hippocampal apoptosis), rendering it unsuitable as a therapeutic approach to chronic spinal injury. Less severe protocols of repetitive acute intermittent hypoxia may elicit plasticity without associated morbidity. Here we demonstrate that daily acute intermittent hypoxia ( $\mathrm{dAIH} ; 10$ episodes per day, $7 \mathrm{~d}$ ) induces motor plasticity in respiratory and nonrespiratory motor behaviors without evidence for associated morbidity. dAIH induces plasticity in spared, spinal pathways to respiratory and nonrespiratory motor neurons, improving respiratory and nonrespiratory (forelimb) motor function in rats with chronic cervical injuries. Functional improvements were persistent and were mirrored by neurochemical changes in proteins that contribute to respiratory motor plasticity after intermittent hypoxia (BDNF and TrkB) within both respiratory and nonrespiratory motor nuclei. Collectively, these studies demonstrate that repetitive acute intermittent hypoxia may be an effective and non-invasive means of improving function in multiple motor systems after chronic spinal injury.
\end{abstract}

\section{Introduction}

Cervical spinal cord injury (SCI) compromises breathing and other motor functions, such as reaching and walking (Frankel et al., 1998). Spinal injuries are usually incomplete, enabling partial spontaneous recovery of respiratory and somatic motor function attributable to plasticity in spared synaptic pathways (Raineteau and Schwab, 2001; Kaegi et al., 2002; Goshgarian, 2003). However, because spontaneous functional recovery is limited, therapeutic strategies are needed to further enhance motor function after chronic SCI. Because limited progress has been made in regenerating spinal pathways (Cafferty et al., 2008; Boulenguez

Received Jun. 9, 2011; revised Dec. 6, 2011; accepted Jan. 14, 2012

Author contributions: M.R.L.-B., G.D.M., and G.S.M. designed research; M.R.L.-B., I.S., G.D.M., J.E.R.W., and M.S.H. performed research; M.R.L.-B., I.S., G.D.M., J.E.R.W., M.S.H., S.V., and G.S.M. analyzed data; M.R.L.-B., I.S., G.D.M., S.V., and G.S.M. wrote the paper.

The Wisconsin work was supported by National Institutes of Health Grants R37 HL69064 (G.S.M.), P01 NS057778 (I.S.), T32 HL07654 (J.E.R.W., M.S.H.), and T32 GM007507 (M.R.L.-B.), and the Craig H. Neilsen Foundation (S.V.). The Saskatchewan work was supported by Natural Sciences and Engineering Research Council of Canada. We thank Bradley Wathen and Dr. Erica Dale for help in preparation of the figures.

*M.R.L.-B., I.S., and G.D.M. contributed equally to this work.

Correspondence should be addressed to Dr. Gordon S. Mitchell, Department of Comparative Biosciences, University of Wisconsin, 2015 Linden Drive, Madison, WI 53706. E-mail: mitchell@svm.vetmed.wisc.edu.

M. R. Lovett-Barr's present address: Department of Pharmacology and Physiology, George Washington University, Washington, DC 20037.

J. E. R. Wilkerson's present address: Department of Neuroscience, University of Texas Southwestern Medical Center, Dallas, TX 75390.

M. S. Hoffman's present address: Department of Medicine, University of lowa, lowa City, IA 52242.

DOI:10.1523/JNEUROSCI.2908-11.2012

Copyright $\odot 2012$ the authors $\quad 0270-6474 / 12 / 323591-10 \$ 15.00 / 0$ and Vinay, 2009), enhancing plasticity in spared neural pathways may be a more achievable goal (Ramer et al., 2000; Blight, 2004).

In uninjured rats, acute intermittent hypoxia $(\mathrm{AIH})$ initiates spinal respiratory plasticity, transiently strengthening pathways to respiratory motor neurons (Mahamed and Mitchell, 2007; MacFarlane et al., 2008). For example, AIH-induced phrenic long-term facilitation is initiated by episodic serotonin release on or near phrenic motor neurons (Baker-Herman and Mitchell, 2002; MacFarlane and Mitchell, 2009), stimulating new synthesis of brain-derived neurotrophic factor (BDNF) and activating its high-affinity receptor (TrkB) (Baker-Herman et al., 2004). Although "downstream" signaling includes ERK-MAP kinase activation (M.S.H. and G.S.M., unpublished observation), it is not known whether long-term facilitation arises from increased motor neuron excitability versus increased synaptic strength (Fuller et al., 2000; Bocchiaro and Feldman, 2004; Mahamed and Mitchell, 2007).

AIH (three 5-min episodes) transiently facilitates crossedspinal synaptic pathways to phrenic motor neurons after $\mathrm{C} 2$ hemisection $\left(\mathrm{C}_{2} \mathrm{HS}\right)$. This effect is greater with increasing time after injury, correlating with initial loss and recovery of serotonergic innervation in the phrenic motor nucleus (Golder and Mitchell, 2005). Thus, intermittent hypoxia may be effective at restoring respiratory function in chronic SCI. Chronic (severe) intermittent hypoxia $(\mathrm{CIH})$ elicit greater and/or more enduring respiratory plasticity; for example, $\mathrm{CIH}$ (72 episodes per night, 7 nights) increases spontaneous and evoked phrenic nerve activity 2 weeks after injury (Fuller et al., 2003). Unfortunately, severe CIH pro- 
tocols cause morbidity, including hypertension (Prabhakar and Kumar, 2004; Sajkov, and McEvoy, 2009), hippocampal cell death, and learning deficits (Gozal and Kheirandish-Gozal, 2007). Here we investigate whether a less intense protocol of repetitive intermittent hypoxia, daily $\mathrm{AIH}$ (dAIH) (Wilkerson and Mitchell, 2009), improves respiratory motor recovery without such comorbidity.

Little information is available concerning the impact of intermittent hypoxia on nonrespiratory motor systems, despite many similarities in limb versus respiratory motor systems (e.g., volitional and automatic control, $\alpha$ motor neurons, serotonergic innervation). Here, we tested the hypotheses that dAIH (1) improves breathing and limb function in rats and (2) elicits similar neurochemical plasticity in respiratory and nonrespiratory motor nuclei.

We report that dAIH (10 episodes per day, $7 \mathrm{~d}$ ) (1) improves the capacity to generate tidal volume $\left(\mathrm{V}_{\mathrm{T}}\right)$ and skilled forelimb function in a horizontal ladder-walking task in rats with cervical spinal injuries and (2) elicits similar plasticity in BDNF and TrkB expression in respiratory and nonrespiratory motor nuclei. Collectively, these results demonstrate that repetitive $\mathrm{AIH}$ induces functional plasticity in multiple spinal motor systems and may be an easy and effective therapeutic strategy to treat motor deficits after SCI.

\section{Materials and Methods}

Assessment of respiratory capacity

Experimental procedures were approved by the University of Wisconsin Institutional Animal Care and Use Committee. Experiments were performed on 3- to 5-month-old male Sprague Dawley (plethysmography experiments: Harlan Colony 217) or Lewis (electrophysiology experiments: Harlan Colony 202a) rats. Animals were housed individually with access to food and water ad libitum.

Spinal surgery: $C_{2} H S$. Buprenorphine $(50 \mu \mathrm{g} / \mathrm{kg})$, carpofen $(5 \mathrm{mg} / \mathrm{kg})$, enrofloxacin $(4 \mathrm{mg} / \mathrm{kg})$, and medetomidine $(100 \mu \mathrm{g} / \mathrm{kg})$ were administered subcutaneously 10-20 min before inducing isoflurane anesthesia in a closed chamber. Rats were intubated, and anesthesia was maintained while rats were mechanically ventilated. A C2 laminectomy and durotomy were performed, and the spinal cord was hemisected just caudal to the C2 dorsal roots as described previously (Fuller et al., 2003, 2006; Golder and Mitchell, 2005); $1 \mathrm{~mm}$ of spinal tissue was aspirated to ensure complete hemisection, and the wounds were sutured closed. Sham rats underwent the same procedures without hemisection. Atipamezole (400 $\mu \mathrm{g} / \mathrm{kg}$ ) was given to reverse medetomidine, the endotracheal tube was removed, and the rats were monitored throughout recovery. Rats received analgesic (buprenorphine, $50 \mu \mathrm{g} / \mathrm{kg}$ ) and anti-inflammatory (carpofen, $5 \mathrm{mg} / \mathrm{kg}$ ) drugs for $2 \mathrm{~d}$ after surgery, with enrofloxacin $(4 \mathrm{mg} / \mathrm{kg})$ given as needed.

$d A I H$. dAIH began 1 week after surgery and lasted for $7 \mathrm{~d}$. Each day, rats were placed in a Plexiglas chamber flushed with a mixture of air/ $\mathrm{N}_{2} / \mathrm{O}_{2}(4 \mathrm{~L} / \mathrm{min})$ to attain continuous normoxia or intermittent hypoxia ( 75 s equilibration; 5 min episodes of $10.5 \% \mathrm{O}_{2} ; 5$ min intervals of $21 \%$ $\mathrm{O}_{2}$ ). After 10 hypoxic episodes, or the equivalent duration or normoxia (110 min), animals were returned to their cages until the next exposure.

Ventilatory measurements in unanesthetized rats. In rats studied $7 \mathrm{~d}$ after dAIH (i.e., $14 \mathrm{~d}$ after injury), whole-body plethysmography (Buxco) was used to measure $V_{T}$, breathing frequency $(f)$, and minute pulmonary ventilation $\left(\dot{V}_{\mathrm{E}}\right)$. After a 30 min acclimation period, baseline ventilation was recorded under normoxic $\left(21 \% \mathrm{O}_{2}\right)$ conditions for $>30 \mathrm{~min}$. Rats were then exposed to $25 \mathrm{~min}$ each of $5 \%$ and $7 \% \mathrm{CO}_{2}$ in $21 \% \mathrm{O}_{2}$ (balance $\mathrm{N}_{2}$ ), followed by $25 \mathrm{~min}$ of $7 \% \mathrm{CO}_{2}$ with hypoxia $\left(10.5 \% \mathrm{O}_{2}\right)$. This procedure enabled an assessment of maximal chemo-reflex stimulated breathing in unanesthetized rats. Gas mixtures flowed continuously through the chamber at $2 \mathrm{~L} / \mathrm{min}$ to prevent $\mathrm{CO}_{2}$ buildup and allow rapid control of inspired gas concentrations. Data were analyzed in $5 \mathrm{~min}$ bins and are presented as means \pm 1 SEM.
Bilateral phrenic nerve recordings in anesthetized rats. Maximal bilateral phrenic motor output and the efficacy of crossed spinal synaptic pathways to phrenic motor neurons were assessed $1 \mathrm{~d}$ after the final dAIH exposure. In these experiments, isoflurane anesthesia was induced in a closed chamber and maintained via nose cone $(2.5-3.5 \%$ isoflurane in $50 \% \mathrm{O}_{2}$, balance $\mathrm{N}_{2}$ ) until a tracheotomy was complete. Rats were then mechanically ventilated $\left[\mathrm{V}_{\mathrm{T}}=2-2.5 \mathrm{ml}\right.$; Fraction of inspired oxygen $\left(\mathrm{FIO}_{2}\right)=0.50$; Rodent Respirator model 682; Harvard Apparatus] with isoflurane anesthesia. The rats were then bilaterally vagotomized, and a femoral artery was catheterized to monitor blood pressure (Gould P23ID) and blood gases (ABL-500; Radiometer). Fluids (lactated Ringer's solution/Hetastarch, $4: 1$ by volume; $2.5 \mathrm{ml} / \mathrm{h}$ ) and any necessary drugs were administered via tail-vein catheter. Phrenic nerves were isolated via a dorsal approach, cut distally, desheathed, and positioned on bipolar silver electrodes submerged in mineral oil. Rats were then converted slowly from isoflurane to urethane anesthesia $(1.6-1.7 \mathrm{mg} / \mathrm{kg})$ and paralyzed with pancuronium bromide $(2.5 \mathrm{mg} / \mathrm{kg}$, i.v.). End-tidal $\mathrm{CO}_{2}$ partial pressure was monitored using a $\mathrm{CO}_{2}$ analyzer with sufficient response time to measure end-tidal $\mathrm{PCO}_{2}$ in rats (Capnoguard; Novametrix Medical Systems), and body temperature was maintained with a temperature-controlled table $\left(37.5 \pm 1^{\circ} \mathrm{C}\right)$.

Nerve signals were amplified (gain of 10,000), filtered (100-10,000 $\mathrm{Hz}$; A-M Systems), rectified, and integrated (time constant, $50 \mathrm{~ms}$; CWE 821 filter; Paynter). Spontaneous inspiratory bursts in both phrenic nerves were digitized, recorded, and analyzed using a WINDAQ data acquisition system (DATAQ Instruments). The $\mathrm{CO}_{2}$ apneic and recruitment thresholds for inspiratory phrenic nerve activity were determined at least $1 \mathrm{~h}$ after conversion to urethane anesthesia. To determine the $\mathrm{CO}_{2}$ apneic threshold, inspired $\mathrm{CO}_{2}$ was decreased until inspiratory phrenic nerve activity ceased; inspired $\mathrm{CO}_{2}$ was then slowly increased until inspiratory activity resumed. The end-tidal $\mathrm{CO}_{2}$ partial pressure at which inspiratory activity resumed is the $\mathrm{CO}_{2}$ recruitment threshold. End-tidal $\mathrm{PCO}_{2}$ was maintained at $2-3 \mathrm{mmHg}$ above the recruitment threshold during baseline measurements $(30-60 \mathrm{~min})$. Three graded respiratory challenges were then imposed: (1) $20 \mathrm{mmHg}$ increase in arterial $\mathrm{CO}_{2}$ partial pressure $\left(\mathrm{PaCO}_{2}\right)$ above baseline; (2) $40 \mathrm{mmHg}$ increase in $\mathrm{PaCO}_{2}$ above baseline; and (3) $40 \mathrm{mmHg}$ increase in $\mathrm{PaCO}_{2}$ combined with hypoxia $\left(\mathrm{PaO}_{2}\right.$ of $\left.\sim 35-45 \mathrm{mmHg} ; \mathrm{FIO}_{2}=0.11 \pm 0.01\right)$. Each challenge lasted 5-7 $\mathrm{min}$, and blood samples were drawn $(0.3 \mathrm{ml})$ during each challenge to confirm $\mathrm{PaCO}_{2}$ and $\mathrm{PaO}_{2}$ levels. Baseline conditions were then restored.

Evoked phrenic nerve recordings were made as described previously (Golder et al., 2008) in hyperventilated rats $\left(\mathrm{PaCO}_{2} \leq 10 \mathrm{mmHg}\right.$ below the apneic $\mathrm{CO}_{2}$ threshold) to prevent spontaneous inspiratory activity. A monopolar tungsten stimulating electrode ( $5 \mathrm{M} \Omega$; A-M Systems) was placed in the ventrolateral funiculus below the dorsal root entry zone on the uninjured side rostral to the lesion. Electrode depth was chosen to maximize the amplitude of short-latency evoked potential ( $\leq 1 \mathrm{~ms})$ on the uninjured side. The ventrolateral funiculus was stimulated with current pulses from 100 to $1500 \mu \mathrm{A}(0.2 \mathrm{~ms}$ duration) to generate a stimulus-response curve (Grass Stimulator model S88 and stimulus isolation unit model PSIU6E; Grass Instruments). Nerve potentials were digitized and analyzed with pClamp software (Molecular Devices).

Data analyses. Data from plethysmography and spontaneous phrenic nerve recording experiments were analyzed with two-way, repeatedmeasures ANOVAs using the Student-Newman-Keuls post hoc test. Evoked potentials on the uninjured side were analyzed with a two-way, repeated-measures ANOVA with Student-Newman-Keuls post hoc test. Evoked potentials on the injured side were analyzed using a $\chi^{2}$ test with a Mann-Whitney post hoc test. Data are presented as mean \pm 1 SEM.

\section{Immunohistochemistry and immunofluorescence}

Immunohistochemistry. Four rat groups $\left(\mathrm{C}_{2} \mathrm{HS}\right.$ or sham surgery treated with normoxia or dAIH, see above; $n=5$ per group) were killed and perfused transcardially with ice-cold $0.01 \mathrm{M}$ PBS, pH 7.4, followed by $4 \%$ buffered paraformaldehyde. Cervical spinal cords were excised, postfixed overnight, and cryoprotected in $30 \%$ sucrose at $4^{\circ} \mathrm{C}$ until the tissues sank. Transverse sections $(40 \mu \mathrm{m})$ of $\mathrm{C} 4$ and $\mathrm{C} 7$ were cut with a freezing microtome (Leica SM 200R). Free-floating sections were washed in $0.1 \mathrm{M}$ 

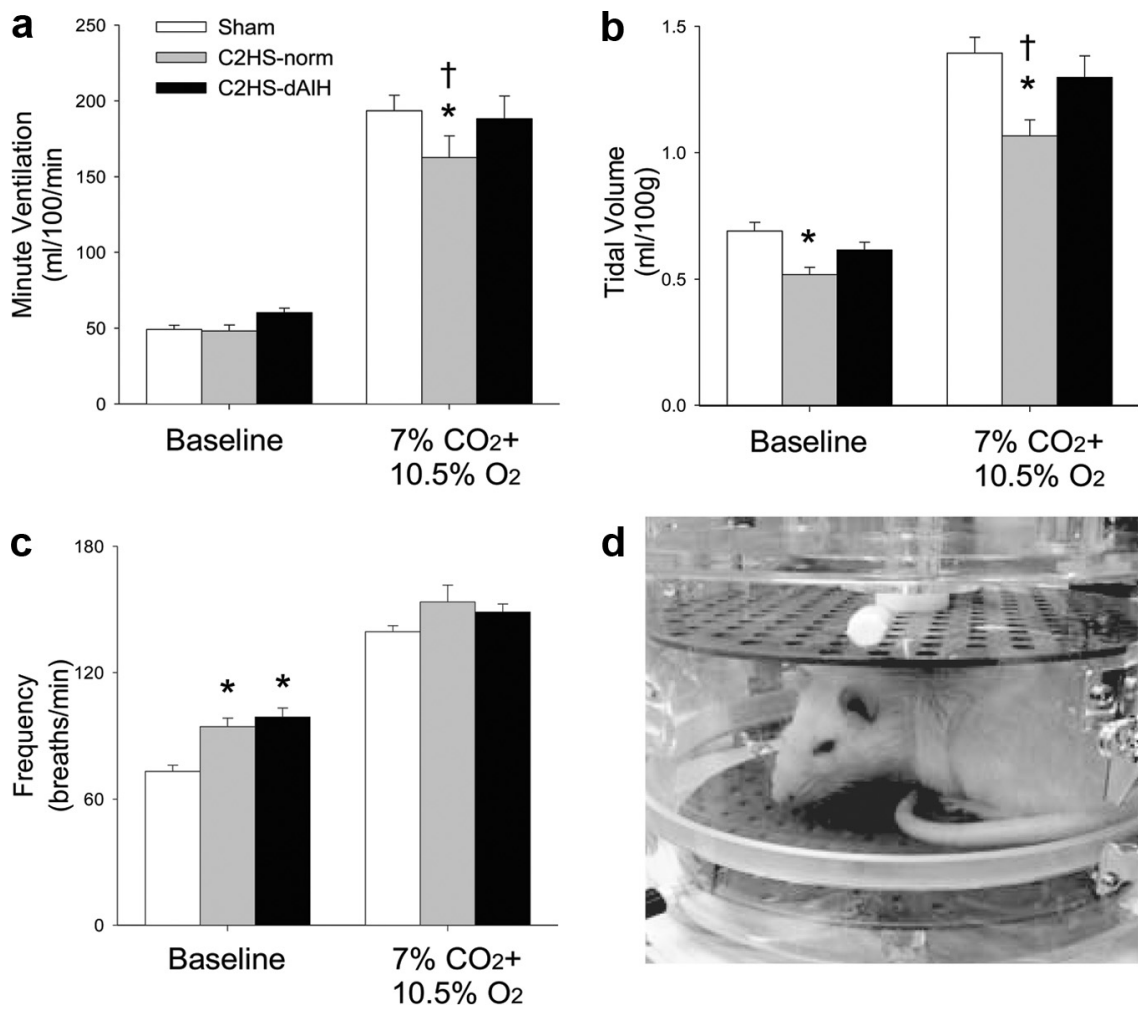

Figure 1. dAlH restores ventilatory capacity in rats with cervical (C2) spinal hemisection. Using whole-body plethysmography $(\boldsymbol{d}), \dot{V}_{\mathrm{E}}(\boldsymbol{a})$ and its components, $\mathrm{V}_{\mathrm{T}}(\boldsymbol{b})$ and breathing frequency $(\boldsymbol{c})$, were assessed during air breathing (baseline) and when breathing an hypercapnic/hypoxic gas mixture $\left(7 \% \mathrm{CO}_{2} / 10.5 \% \mathrm{O}_{2}\right)$ to assess ventilatory capacity. Neither $\mathrm{C}_{2} \mathrm{HS}(\mathrm{C} 2 \mathrm{HS}$-norm; gray bars) nor dAIH in hemisected rats ( $\left(2 \mathrm{HS}-\mathrm{dAlH}\right.$; black bars) affected $\dot{\mathrm{V}}_{\mathrm{E}}$ during baseline conditions. However, $\mathrm{C}_{2} \mathrm{HS}$ decreased ventilatory capacity during maximal chemoreceptor stimulation versus sham rats $(p=0.04)$. dAIH increased ventilatory capacity after $\mathrm{C}_{2} \mathrm{HS}$, restoring ventilation during maximal chemoreceptor stimulation to normal levels (not significantly different vs sham rats). $\mathrm{C}_{2} \mathrm{HS}$ decreased $\mathrm{V}_{\mathrm{T}}$ during baseline $(25 \% ; p=0.036)$ and chemoreceptor-stimulated breathing $(22 \% ; p=0.005)$; $\mathrm{dAIH}$ restored $70 \%$ of this lost capacity to increase $\mathrm{V}_{\mathrm{T}}$ during chemoreceptor stimulation in $\mathrm{C}_{2} \mathrm{HS}$ rats $(p=0.028)$. Breathing frequency was increased by $\mathrm{C}_{2} \mathrm{HS}$ during baseline conditions $(29 \% ; p<0.001)$, although not during chemoreceptor stimulation; frequency was not affected by dAlH in either condition. ${ }^{*} p<0.05$ versus sham animals; ${ }^{\dagger} p<0.05$ versus $\mathrm{C} 2 \mathrm{HS}$-dAIH.

Tris-buffered saline with $0.1 \%$ Triton X-100 (TBS-Tx; three times for 5 min) and incubated in TBS containing $1 \% \mathrm{H}_{2} \mathrm{O}_{2}$ for $30 \mathrm{~min}$. After washing (three times for $5 \mathrm{~min}$ ) in TBS-Tx, tissues were blocked with 5\% normal goat serum at room temperature (RT) for $60 \mathrm{~min}$. Staining was performed by incubating sections with rabbit polyclonal anti-BDNF $(\mathrm{N}-$ 20, sc-546; 1:1000; Santa Cruz Biotechnology), rabbit anti-TrkB (H-181, sc-8316; 1:1000; Santa Cruz Biotechnology), or rabbit anti-phosphoTrkB (1:1000; courtesy of Dr. Moses Chao, New York University, New York, NY) at $4^{\circ} \mathrm{C}$ overnight. The sections were washed in TBS-Tx and incubated in biotinylated secondary goat anti-rabbit antibody (1:1000; Vector Laboratories). Conjugation with avidin-biotin complex (Vecstatin Elite ABC kit; Vector Laboratories) was followed by visualization with 3,3'-diaminobenzidine hydrogen peroxidase (Vector Laboratories) according to the instruction of the manufacturer. Sections were then washed in TBS, placed on gelatin-coated slides, dried, dehydrated in a graded alcohol series, cleared with xylenes, and mounted with Eukitt mounting medium (Electron Microscope Sciences). All images were captured and analyzed with a digital camera (SPOT II; Diagnostic Instruments). Photomicrographs were created with Adobe Photoshop software (Adobe Systems). All images received equivalent adjustments to tone scale, gamma, and sharpness. Negative controls were performed by omitting the primary or secondary antibodies; in addition, we preabsorbed the primary BDNF antibody with a fivefold (by concentration) excess of a specific blocking peptide (sc-546 P; Santa Cruz Biotechnology).

Immunofluorescence. Gliosis in hippocampal sections was assessed using antibodies targeting a marker for astrocytes (GFAP; 1:1000; Millipore Bioscience Research Reagents) or microglia (OX-42; 1:500; AbD Serotec). Sections were incubated at $4^{\circ} \mathrm{C}$ overnight and, after washing with
TBS-Tx (three times for $5 \mathrm{~min}$ ), were incubated in conjugated goat anti-rabbit red fluorescent Alexa Fluor 495 (1:200; Invitrogen) at RT for $60 \mathrm{~min}$. Tissue sections were mounted on glass using anti-fade solution (Prolong Gold antifade reagent; Invitrogen) and examined using an epifluorescence microscope (Nikon).

TUNEL assay. Apoptotic cell death in hippocampal sections was assessed using TUNEL with the In Situ Cell Death Detection Kit (Roche Molecular Biochemical) according to the instructions of the manufacturer. Briefly, hippocampal sections $(40 \mu \mathrm{m})$ were permeabilized with $1 \%$ Proteinase $\mathrm{K}$ (in $50 \mathrm{~mm}$ Tris/5 mM EDTA buffer) for $15 \mathrm{~min}$, rinsed with PBS, and incubated in TUNEL reaction mixture for $1 \mathrm{~h}$ at $37^{\circ} \mathrm{C}$. Samples were rinsed, mounted in anti-fade solution (Invitrogen), and analyzed with the epifluorescence microscope. TUNEL (red)-stained tissues were also exposed to antiNeuN (green) to identify any apoptotic neurons. Cresyl violet staining was used to identify any nonspecific dAIH-induced cell death in separate hippocampal sections.

Quantification and statistical analysis. Sections were numbered sequentially, and every eighth section was selected for immunohistochemistry. Thus, approximately six sections from each animal at each segmental level were used in this study. The phrenic motor nucleus was identified as a cluster of large neurons in the mediolateral C4 ventral horn (described by Boulenguez et al., 2007; Mantilla et al., 2009). Digital photomicrographs of immunoreactive labeling in the region of phrenic motor neurons were taken with a $20 \times$ objective lens (SPOT II; Diagnostic Instruments). Densitometry was performed by circumscribing the phrenic motor nucleus based on their location and large size in the medial-lateral-ventral aspect of the C4 ventral horn (Boulenguez et al., 2007; Mantilla et al., 2009; Dale-Nagle et al., 2011). A similar procedure was used in the $C 7$ ventral horn, although the area of interest was centered on spinal lamina 9 (Paxinos and Watson, 1997). The intensity of BDNF-, TrkB-, and phospho-TrkBimmunoreactive staining in the defined area of interest was quantified using NIH Image J software (National Institutes of Health; http://rsb.info.nih.gov/ij). Images were converted to eight-bit resolution, and the threshold was set between 120 and 160 during all analyses. The optical density (OD) was measured within circumscribed labeled motor neurons and expressed as an averaged of OD per unit area for each individual cell. For each cell, the OD of BDNF, TrkB, and phospho-TrkB immunoreactivity was expressed as a fraction of the average OD of sham animals. Thus, the mean OD in the sham rats is expected to be 1.0, with variance that reflects variation among the group. Average labeling intensity was compared between treatment groups (each $n=5$ ) using one-way ANOVA followed by Fisher's LSD post hoc test (SigmaStat 2.03; SPSS). The numbers of GFAP-, OX-42-, TUNEL- and Nissl-positive cells in the hippocampus (C2 subfield) were counted manually, and normoxia versus dAIH-treated rats were compared using a $t$ test. Differences were considered significant if $p<0.05$. All values are expressed as mean \pm 1 SEM.

\section{Ladder-walking performance in rats}

Fourteen adult male Lewis rats (body mass, 350-400 g) obtained from Charles River Laboratories were used in this experiment. The rats were housed as pairs, and the cages were kept inside a $12 \mathrm{~h}$ light/dark cycle controlled room at the Animal Care Facility, Western College of Veterinary Medicine at the University of Saskatchewan. The rats were cared for 
according to the guidelines prescribed by the Canadian Council on Animal Care. Rats were conditioned daily to repeatedly cross a horizontal runway, measuring $182 \times 20 \mathrm{~cm}$ for a food reward. After they were conditioned to trot on the runway, they were conditioned to run back and forth repeatedly over a horizontal ladder (see below) for a food reward. After $\sim 2-3$ weeks, all animals would cross the ladder repeatedly without hesitation.

Spinal surgery (partial $\mathrm{C}_{2} \mathrm{HS}$ ). Rats were premedicated with an anti-cholinergic drug, glycopyrrolate $(0.03 \mathrm{mg} / \mathrm{ml}$, s.c.; Sabex $)$ and a mixture of medetomidine hydrochloride $(0.3$ mg/kg, i.p.; Damitor; Novartis Animal Health) and buprenorphine $(0.05 \mathrm{mg} / \mathrm{kg}$, i.p.; Buprenex; Reckitt Benckiser Pharmaceuticals). A surgical plane of anesthesia was induced and maintained with isoflurane administered by mask. After anesthetic induction, a unilateral dorsolateral funiculotomy was performed as described previously (Webb and Muir, 2003; Muir et al., 2007). Briefly, the skin and muscles over the dorsal cervical spinal cord were incised and bluntly dissected to expose the dorsal spinous processes and spinal laminae of the second and third cervical vertebrae. A laminectomy and durotomy were performed to expose the spinal cord. Using the entry site of the dorsal roots as a landmark, the dorsolateral funiculus at the level of the third cervical spinal segment was transected unilaterally using a modified 25-gauge bevel-tipped needle. An autologous fat graft obtained near the surgical site was placed over the laminectomy site, and the muscles and skin were closed.

$d A I H$. Four weeks after surgery, rats were randomly assigned to receive either $\mathrm{dAIH}$ or normoxia. The day before treatment onset, rats were acclimated to custom-made Plexiglas chambers $(1$ rat per chamber; $30 \times 17 \times 12$ $\mathrm{cm})$ under normoxic conditions $\left(\mathrm{FIO}_{2}=\right.$ $0.21 \pm 0.005)$. For the next $7 \mathrm{~d}$, rats were placed into the Plexiglas chambers and exposed daily to $\mathrm{AIH}(n=7)$ or normoxia $(n=7)$. As in the previous groups, dAIH consisted of 105 -min episodes of hypoxia $\left(\mathrm{FIO}_{2}=0.11\right)$ interspersed with 5 -min normoxic intervals. Sham-treated rats were in the chambers for an equivalent period of time under continually normoxic conditions. Oxygen levels were continuously monitored (AX300-1 Portable Oxygen Analyzer; Teledyne Analytical Instruments). After acclimation to the chambers, both dAIH- and normoxiatreated rats would rest quietly or sleep throughout the exposure period.

Behavioral assessment of skilled locomotion: horizontal ladder test. The horizontal ladder was used to assess the skilled locomotor ability of the rats as described previously (Poulton and Muir, 2005; Muir et al., 2007; Kanagal and Muir, 2009). Briefly, a ladder consisting of 2-mm-diameter metallic rungs placed $2 \mathrm{~cm}$ apart was used. The ladder was placed above a $45^{\circ}$ angled mirror so that rats could be videotaped simultaneously from both a lateral and ventral view. At each session, a minimum of 10 runs were recorded from each rat using a VHS camera. Videotapes were analyzed field by field ( 60 fields/s), and the number of footfalls by each limb were counted as the rats walked over the rungs. Data from only one complete stride was sampled from each pass to satisfy statistical requirements for independence between multiple measurements from an individual limb. Data from at least 10 passes from each rat were averaged for each day of data collection. Data were expressed as number of correct steps per total steps for each limb per rat. Assessment of ladder locomotion was performed before surgery and at 4 weeks after surgery on the day before dAIH treatment. Ladder locomotion was also assessed $1 \mathrm{~h}$ after versus sham rats.

\section{b Uninjured Side}
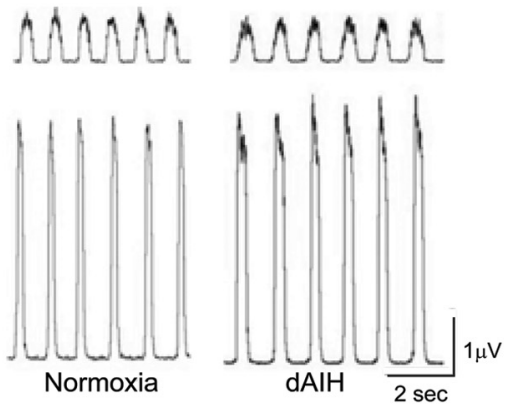

d Injured Side (evoked)

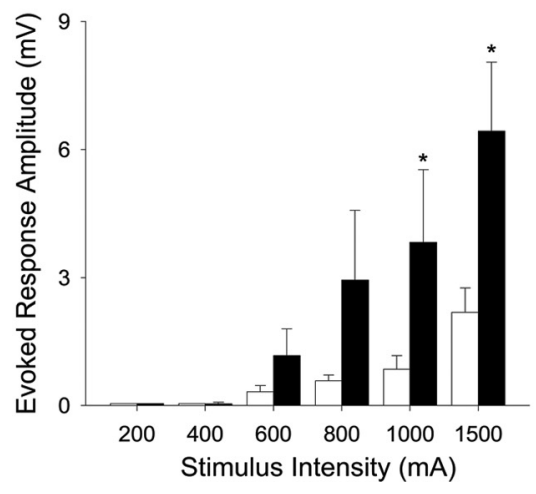
Stimulus Intensity (mA)

Figure 2. dAlH strengthens spontaneous phrenic nerve activity and crossed-spinal synaptic pathways to phrenic motor neurons after $\mathrm{C}_{2} \mathrm{HS}$. In $\boldsymbol{a}$ and $\boldsymbol{b}$, integrated phrenic neurograms are shown to illustrate spontaneous phrenic nerve activity before and after AlH in rats with $\mathrm{C}_{2} \mathrm{HS}$. Whereas $\mathrm{dAlH}$ increased spontaneous phrenic activity ipsilateral to hemisection during baseline and

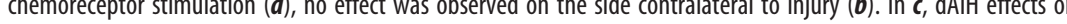
responses evoked by electrical stimulation of the contralateral ventrolateral funiculus are shown. The amplitude of evoked phrenic responses is greater in dAlH-treated (filled bars) versus normoxia-treated (open bars) rats at all stimulus intensities. ${ }^{*} p<0.05$

AIH treatment on each exposure day and again 1 and 3 weeks after the final AIH treatment.

Analyses. Group data were averaged and expressed as means \pm 1 SEM. Groups were compared using a two-way, repeated-measures ANOVA (SigmaStat; Systat Software). Post hoc analysis was performed using the Holm-Sidak method with $p<0.05$ considered significant.

\section{Results}

dAIH does not affect breathing in uninjured rats

dAIH had no significant effects on breathing in uninjured rats. There were no differences between $\mathrm{V}_{\mathrm{T}}$ in normoxia-treated and dAIH-treated sham rats under any test condition $(p>0.05$; Fig. $1 b)$. Because breathing frequency was also unaffected by dAIH in sham rats $(p>0.05$; Fig. $1 c)$, dAIH had no effect on $\dot{V}_{\mathrm{E}}(p>0.05$; Fig. 1a). Finally, dAIH had no effect on mean inspiratory flow $\left(\mathrm{V}_{\mathrm{T}} / \mathrm{Ti}\right.$, an indicator of inspiratory drive) under any condition in sham rats $(p>0.05$; data not shown). In subsequent analyses, normoxia-treated and $\mathrm{dAIH}$-treated shams were combined into a single sham group.

\section{$\mathrm{C}_{2}$ HS impairs breathing capacity}

As reported previously (Fuller et al., 2006), $\mathrm{C}_{2} \mathrm{HS}$ reduces VT $(p<0.05$; Fig. $1 b)$; rats compensate for reduced VT by elevating breathing frequency $(p<0.05$; Fig. $1 c)$. Thus, $\mathrm{C}_{2} \mathrm{HS}$ shifts the breathing pattern $(p<0.05)$ but has little effect on $\dot{\mathrm{V}}_{\mathrm{E}}$ or mean 
Table 1. Arterial $\mathrm{CO}_{2}$ partial pressures $(\mathrm{mmHg})$ during phrenic nerve recordings

\begin{tabular}{llllll}
\hline Treatment & $n$ & Baseline & Hypercapnia 1 & Hypercapnia 2 & $\begin{array}{l}\text { Hypoxia and } \\
\text { hypercapnia }\end{array}$ \\
\hline $\mathrm{C}_{2}$ HS-dAlH & 6 & $45 \pm 1$ & $65 \pm 1$ & $85 \pm 1$ & $86 \pm 1$ \\
$\mathrm{C}_{2}$ HS-normoxia & 7 & $43 \pm 1$ & $62 \pm 1$ & $84 \pm 2$ & $83 \pm 1$ \\
\hline
\end{tabular}

Mean values \pm 1 SEM.

Table 2. Arterial $\mathrm{O}_{2}$ partial pressures $(\mathrm{mmHg}$ ) during phrenic nerve recordings

\begin{tabular}{llllll}
\hline Treatment & $n$ & Baseline & Hypercapnia 1 & Hypercapnia 2 & $\begin{array}{l}\text { Hypoxia and } \\
\text { hypercapnia }\end{array}$ \\
\hline C $_{2}$ HS - dAIH & 6 & $270 \pm 10$ & $253 \pm 19$ & $252 \pm 24$ & $46 \pm 1$ \\
C $_{2}$ HS-normoxia & 7 & $247 \pm 24$ & $264 \pm 21$ & $275 \pm 8$ & $45 \pm 1$ \\
\hline
\end{tabular}

Mean values \pm 1 SEM.

inspiratory flow during baseline conditions breathing room air $(p>0.05$; Fig. 1$)$. Conversely, $\mathrm{C}_{2} \mathrm{HS}$ rats reveal $\dot{\mathrm{V}}_{\mathrm{E}}$ deficits when challenged with combined hypercapnia/hypoxia $(p<0.05$; Fig. $1 a$ ), confirming that $\mathrm{C}_{2} \mathrm{HS}$ causes a respiratory deficit characterized by the inability to respond to respiratory challenges (Fuller et al., 2006). This deficit in breathing capacity is characterized by a diminished ability to increase $\mathrm{V}_{\mathrm{T}}$ during hypercapnia/hypoxia $(p<0.05$; Fig. $1 b)$, with minimal effect on the ability to increase breathing frequency (Fig. 1c). A decreased ability to increase $\mathrm{V}_{T}$ is consistent with disrupted bulbo-spinal synaptic inputs to respiratory motor neurons below a hemisection.

\section{dAIH restores breathing capacity after $\mathrm{C}_{2} \mathrm{HS}$}

dAIH improves the ability to increase $\dot{\mathrm{V}}_{\mathrm{E}}$ in $\mathrm{C}_{2} \mathrm{HS}$ rats versus normoxia-treated $\mathrm{C}_{2} \mathrm{HS}$ rats $(p<0.05$; Fig. $1 a)$. In fact, dAIH treatment of $\mathrm{C}_{2} \mathrm{HS}$ rats restores $\dot{\mathrm{V}}_{\mathrm{E}}$ during hypercapnia/hypoxia so that is no longer significantly reduced versus uninjured controls $\left(p>0.05\right.$; Fig. 1a). This increase in $\dot{V}_{\mathrm{E}}$ is achieved primarily by increased $V_{T}$ (Fig. 1b) with minimal contribution from changes in frequency (Fig. 1c). $\mathrm{V}_{\mathrm{T}}$ in dAIH-treated $\mathrm{C}_{2} \mathrm{HS}$ rats is increased versus normoxia-treated $\mathrm{C}_{2} \mathrm{HS}$ rats during maximal chemoreceptor stimulation ( $p<0.05$; Fig. $1 b)$. Conversely, although $\mathrm{V}_{\mathrm{T}}$ tended to be lower than sham rats during hypercapnia/hypoxia, it was no longer significantly reduced $(p>0.05)$. Thus, the capacity to generate increased $\mathrm{V}_{\mathrm{T}}$ was substantially increased by dAIH. Frequency was similar in normoxia- and dAIHtreated $\mathrm{C}_{2} \mathrm{HS}$ rats $(p>0.05$; Fig. $1 c)$ but remained elevated versus uninjured rats $(p<0.05$; Fig. $1 c)$. Mean inspiratory flow was unaffected by dAIH ( $p>0.05$; data not shown). Thus, dAIH restores $\dot{V}_{\mathrm{E}}$ to uninjured levels for $\geq 1 \mathrm{~d}$ after dAIH, primarily by a restored capacity to increase $V_{T}$ during maximal chemoreceptor stimulation. Collectively, these results demonstrated remarkable restoration of breathing capacity but with a persistent alteration in the pattern of breathing characteristic of $\mathrm{C}_{2} \mathrm{HS}$ rats.

\section{dAIH increases phrenic motor output after $\mathrm{C}_{2} \mathrm{HS}$}

In anesthetized, paralyzed and ventilated rats with $\mathrm{C}_{2} \mathrm{HS}$, blood gas regulation was comparable between $\mathrm{dAIH}-$ and normoxiatreated rats (Tables 1,2), ensuring that observed differences in spontaneous phrenic activity are not attributable to differences in chemoreceptor feedback. During baseline conditions, barely discernable spontaneous phrenic nerve bursts were observed on the injured side in three of six normoxia-treated rats. In contrast, robust phrenic bursts were observed on the injured side in four of five dAIH-treated rats during baseline conditions (Fig. 2a). dAIH increased spontaneous phrenic activity on the injured side at all levels of chemo-reflex drive up to the maximal hypercapnic/hypoxic response (all $p<0.001$; Fig. $2 c$ ), demonstrating that dAIH
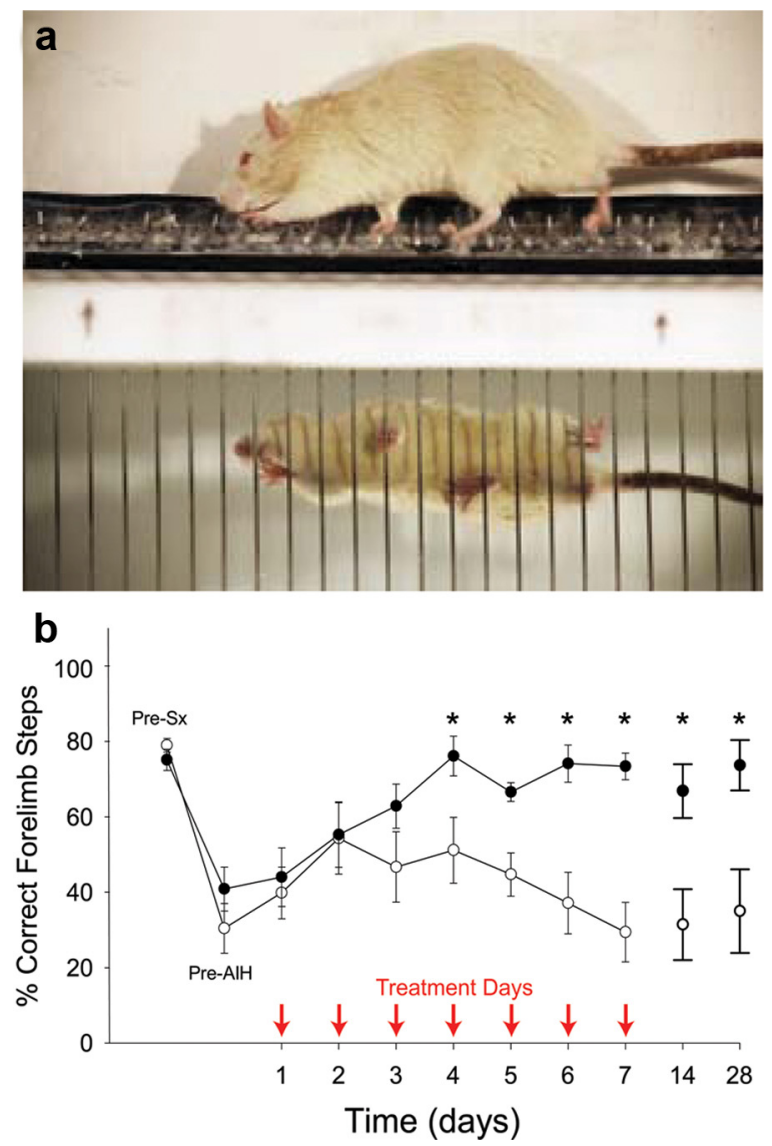

Figure 3. Forelimb ladder-stepping ability improves after dAlH in rats with incomplete cervical spinal injuries. In $\boldsymbol{a}$, forelimb ladder-stepping performance was assessed in spinally injured rats with and without dAIH treatment. In $\boldsymbol{b}$, rats exposed to dAlH beginning 4 weeks after transection of the dorsolateral spinal funiculus at C3 (filled circles; $n=7$ ) made significantly fewer errors with the impaired forelimb beginning at day 4 of treatment versus injured rats exposed to normoxia only (sham, open circles, $n=7$ ). Treatment days are indicated by arrows. Full functional recovery lasted $\geq 3$ weeks after $\mathrm{dAlH}$ treatment. Performance was measured as the number of correct steps/total steps while walking over a horizontal ladder; measurements were made before surgery (Pre-Sx), 4 weeks after surgery but before dAIH (Pre-AlH), $1 \mathrm{~h}$ after AlH on each treatment day (arrows), and at 7 and $21 \mathrm{~d}$ after the AlH treatments had ended. ${ }^{*} p<0.05$ versus sham rats at the same time point.

partially restored spontaneous phrenic nerve activity below the injury. Robust spontaneous phrenic nerve bursts were observed in all rats on the uninjured side (Fig. $2 b$ ), although these spontaneous bursts did not differ between $\mathrm{dAIH}$ - and normoxia-treated rats during baseline conditions ( $p>0.05$; Fig. $2 b$ ). Conversely, $\mathrm{dAIH}$ attenuated phrenic burst amplitude during hypercapnia or hypercapnia with hypoxia on the uninjured side $(p<0.05$; data not shown), possibly reflecting compensation for the increased diaphragm function on the injured side.

Spinal plasticity below the site of injury was demonstrated by short-latency $(<1 \mathrm{~ms})$ evoked potentials elicited via electrical stimulation of the intact ventrolateral funiculus (contralateral to injury) (Fuller et al., 2003; Golder and Mitchell, 2005). Stimulus threshold $(p>0.05)$ and onset latency $(p>0.05)$ of evoked, crossed-phrenic responses were unaffected by dAIH (data not shown). On the uninjured side, the short-latency ( $<1 \mathrm{~ms}$ ) evoked response amplitude was greater in $\mathrm{dAIH}$ - versus normoxiatreated rats $(p<0.05$; data not shown). On the injured side, only one of five normoxia-treated rats exhibited a short-latency evoked crossed-phrenic response; however, short-latency crossedspinal responses were observed in all five dAIH-treated rats 

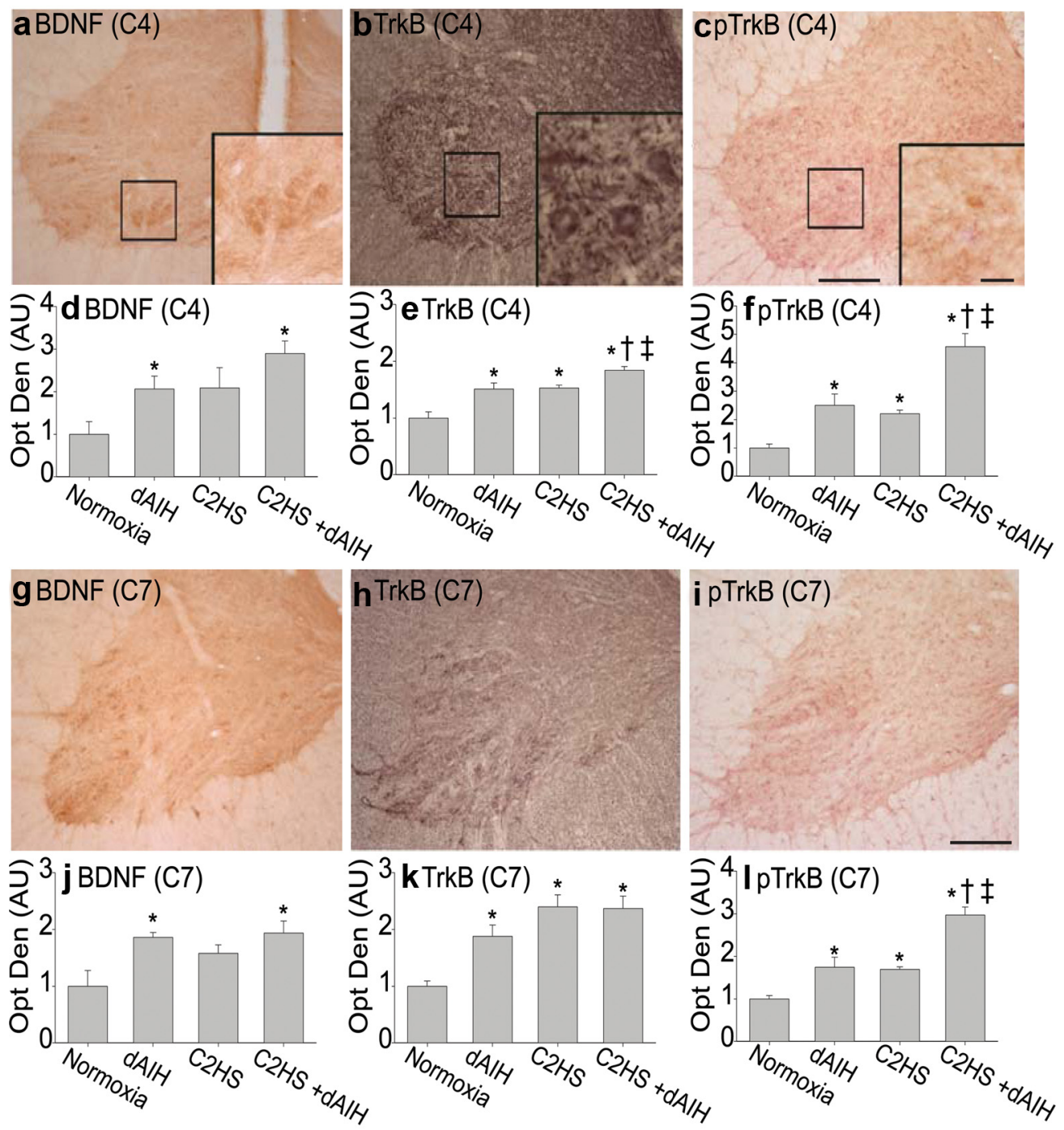

Figure 4. $\mathrm{dAlH}$ and $\mathrm{C}_{2} \mathrm{HS}$ upregulate BDNF, TrkB, and phospho-TrkB in $\mathrm{C} 4$ and $\mathrm{C} 7$ motor nuclei. In $\boldsymbol{a}-\boldsymbol{c}$, examples of BDNF, TrkB, and phosphorylated TrkB (pTrkB) immunoreactivity are shown in the region of the phrenic motor nucleus ( $(4)$ from rats with dAIH plus $\mathrm{C}_{2} \mathrm{HS}(\mathrm{C} 2 \mathrm{HS}+\mathrm{dAIH})$. Other conditions are not shown because of space limitations. Boxes indicate the region of the phrenic motor nucleus in which densitometry was performed; higher-magnification images from this region are in the bottom right corner of these images. Scale bars: lower magnification, $200 \mu \mathrm{m}$; higher magnification, $50 \mu \mathrm{m}$ (same scale in all panels). In $\boldsymbol{d}-\boldsymbol{f}$, summaries of densitometry at (4 are provided for each protein in rats from the following groups (each $n=5$ ): (1) sham-operated rats

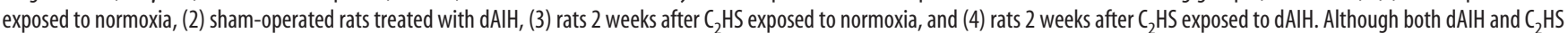
tended to increase BDNF, their combined effect was not greater than either treatment alone. TrkB and phospho-TrkB were increased by dAlH and $\mathrm{C}_{2} \mathrm{HS}$, and their combined effect was greater than either treatment alone (particularly pTrkB). Greater TrkB phosphorylation suggests activation of signaling pathways that underlie phrenic motor plasticity. In $\boldsymbol{g}$ - $\boldsymbol{i}$, examples of BDNF, TrkB, and phosphorylated TrkB immunoreactivity are shown in the $\mathrm{C} 7$ ventral horn from rats with dAIH plus $\mathrm{C}_{2} \mathrm{HS}$ ( $(2 \mathrm{HS}+\mathrm{dAIH})$. Scale bar: $200 \mu \mathrm{m}$. Inj-I, summaries of densitometry at $\mathrm{C} 7$ are provided for the following groups: (1) sham-operated rats exposed to normoxia, (2) sham-operated rats treated with dAlH, (3) rats 2 weeks after $\mathrm{C}_{2} \mathrm{HS}$ exposed to normoxia, and (4) rats 2 weeks after $\mathrm{C}_{2} \mathrm{HS}$ exposed to dAIH. Densitometry was performed in spinal lamina 9 . Results from $\mathrm{C}_{7}$ are strikingly similar to $\mathrm{C} 4$, indicating that $\mathrm{AAIH}$ and $\mathrm{C}_{2} \mathrm{HS}$ elicit similar effects in spinal respiratory and somatic motor nuclei. Data are means \pm 1 SEM. ${ }^{*} p<0.05$ versus normoxia; ${ }^{\dagger} p<0.05$ versus $\mathrm{dAIH} ;{ }^{\ddagger} p<0.05$ versus $\mathrm{C}_{2} \mathrm{HS}$.

$(p<0.01)$. Because dAIH increased the amplitude of evoked crossed-spinal responses ( $p<0.05$; Fig. $2 d$ ), it strengthens crossed-spinal pathways to phrenic motor neurons.

\section{dAIH improves horizontal ladder walking in injured rats}

dAIH elicited nearly complete and prolonged ( $>3$ weeks) recovery of forelimb function in the horizontal ladder-walking task. Before injury, all rats crossed the ladder successfully with $\sim 20 \%$ foot slips (Fig. 3b). Four weeks after injury, significantly more errors were made with the impaired forelimb, slipping $>60 \%$ of the time (Fig. 3b). Rats receiving normoxia and ladder walking beginning 4 weeks after injury did not improve significantly from this level, either during the 1 week period of daily ladder walking or in the 3 weeks after this task. In contrast, rats receiving $\mathrm{dAIH}$ before daily ladder walking rapidly improved and made only $30 \%$ foot-slip errors by the fourth treatment day (Fig. 3b). dAIHtreated rats had significantly fewer foot-slip errors versus predAIH performance $(p<0.001)$ and normoxia-treated animals beyond treatment day $4(p<0.005)$. After the fourth day of treatment, ladder-walking performance was no longer diminished relative to preinjury control measurements $(p>0.05)$; this same level of recovery was still apparent 1 and 3 weeks after dAIH (Fig. 3b).

\section{dAIH increases BDNF, TrkB, and phosphorylated TrkB in motor nuclei}

Constitutive BDNF staining is weak in presumptive motor neurons at both $\mathrm{C} 4$ and $\mathrm{C} 7$ (data not shown). Robust constitutive 

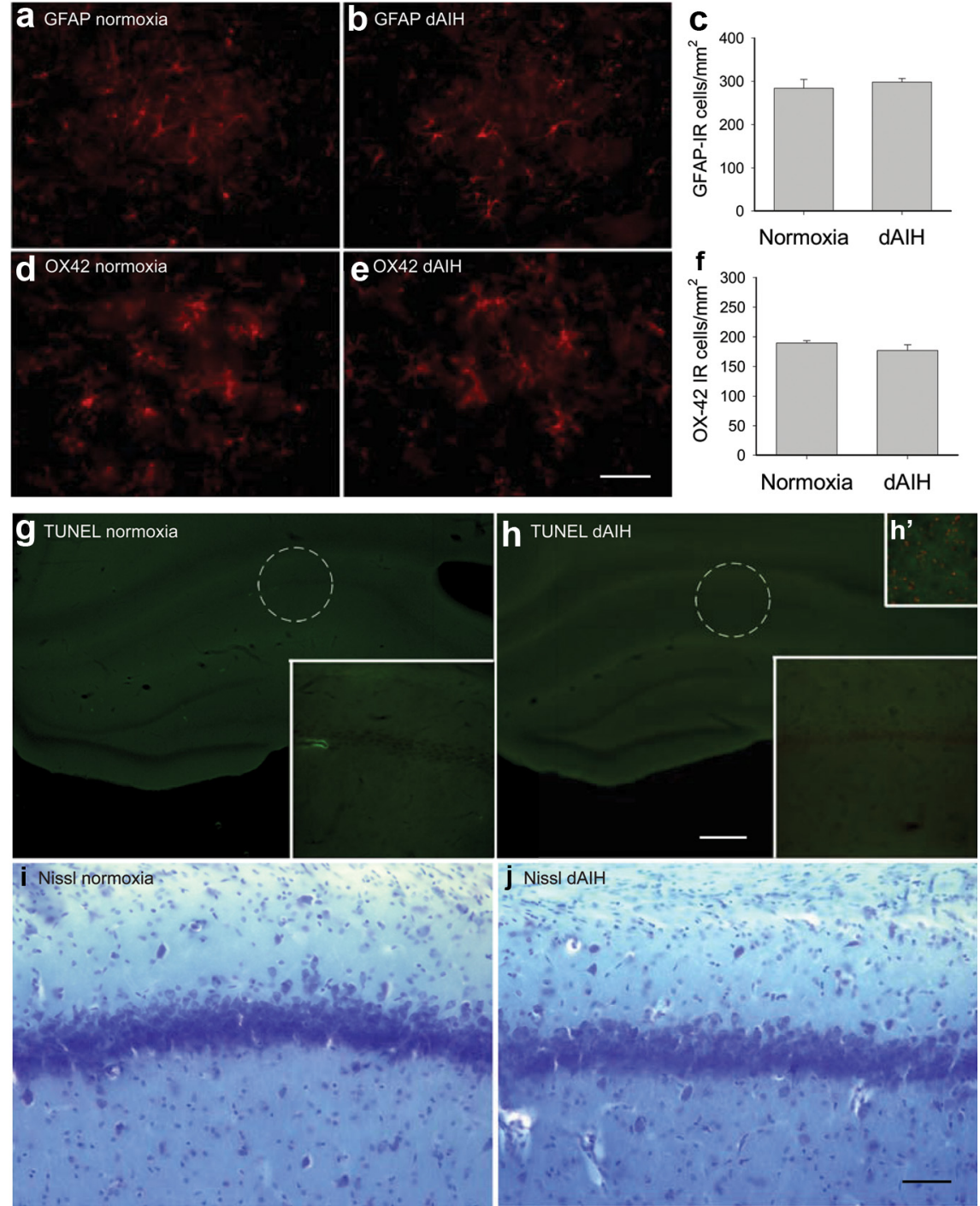

Figure 5. dAlH does not induce hippocampal gliosis or neuron death. Because CIH induces CNS inflammation and hippocampal cell death, we determined whether the more modest $(\mathrm{dAIH})$ protocol elicits similar adverse effects. There was no evidence for reactive astrocytes (GFAP positive; $\boldsymbol{a}-\boldsymbol{c}$ ) or microglia (OX-42 positive; $\boldsymbol{d}-\boldsymbol{f}$ ) after dAlH. Cell counts reveal that the density of GFAP-positive ( $\boldsymbol{c}$ ) and 0X-42 positive $(\boldsymbol{f})$ cells was unaffected by dAIH ( $n=5$ per group), and there were no obvious increases in the intensity of either cell marker ( $\boldsymbol{a}$ vs $\boldsymbol{b} ; \boldsymbol{d}$ vs $\boldsymbol{e}$ ). Furthermore, there was no evidence for dAlH-induced hippocampal apoptosis $(\boldsymbol{g}, \boldsymbol{h})$ or nonspecific cell death $(\boldsymbol{i}, \boldsymbol{j})$ based on TUNEL or cresyl violet staining, respectively. $\boldsymbol{h}^{\prime}$ is a positive control for TUNEL staining taken from the cortex after middle cerebral artery occlusion in a rat. Magnified images of the CA2 hippocampal subfield from control and $\mathrm{dAlH}$-treated rats are presented in the bottom right corners of $\boldsymbol{g}$ and $\boldsymbol{h}$. Some sections were stained with cresyl violet to enable morphological assessment $(\boldsymbol{i}, \boldsymbol{j})$. Data in c and $\boldsymbol{f}$ are means \pm 1 SEM. Scale bars: $\boldsymbol{a}, \boldsymbol{b}, \boldsymbol{d}, \boldsymbol{e}, 50 \mu \mathrm{m} ; \boldsymbol{g}, \boldsymbol{h}, 500 \mu \mathrm{m} ; \boldsymbol{i}, \boldsymbol{j}, 100 \mu \mathrm{m}$.

TrkB staining is observed in presumptive motor neurons in both regions, but phospho-TrkB is scarcely detectable in motor neurons of either segment in sham rats.

$\mathrm{dAIH}(p=0.047)$ and dAIH plus $\mathrm{C}_{2} \mathrm{HS}(p=0.002)$ increased BDNF immunoreactivity in the region of the phrenic motor nucleus (Fig. $4 d$ ), but an apparent increase after $\mathrm{C}_{2} \mathrm{HS}$ alone did not attain statistical significance $(p=0.08)$. Treatment groups did not differ significantly (Fig. $4 d$ ). TrkB labeling followed a similar pattern; dAIH $(p<0.001), \mathrm{C}_{2} \mathrm{HS}(p<0.001)$, and $\mathrm{C}_{2} \mathrm{HS}$ plus dAIH $(p<0.001)$ increased TrkB immunoreactivity, although, in this case, the combined effect was greater than either dAIH $(p=0.025)$ or $\mathrm{C}_{2} \mathrm{HS}$ alone $(p=0.033$; Fig. $4 e)$. dAIH $(p=$ $0.004), \mathrm{C}_{2} \mathrm{HS}(p=0.017)$, and $\mathrm{C}_{2} \mathrm{HS}$ plus dAIH $(p<0.001)$ also increased phospho-TrkB immunoreactivity (Fig. $4 f$ ), and the

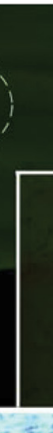

combined effect was greater than $\mathrm{dAIH}$ $(p<0.001)$ or $\mathrm{C}_{2} \mathrm{HS}$ alone $(p<0.001)$.

At C7, labeling within lamina 9 exhibited strikingly similar results to the phrenic motor nucleus. dAIH $(p=0.006)$ and $\mathrm{dAIH}$ plus $\mathrm{C}_{2} \mathrm{HS}(p=0.005)$ increased BDNF immunoreactivity, but an apparent increase after $\mathrm{C}_{2} \mathrm{HS}$ alone was not significant $(p=0.059)$; there were no differences among treatment groups (Fig. $4 j)$. dAIH $(p=0.004), \mathrm{C}_{2} \mathrm{HS}(p<0.001)$, and $\mathrm{C}_{2} \mathrm{HS}$ plus dAIH $(p<0.001)$ increased TrkB immunoreactivity, but this effect did not differ among groups $(p>$ 0.05; Fig. $4 k)$. dAIH $(p=0.014), \mathrm{C}_{2} \mathrm{HS}$ $(p<0.034)$, and $\mathrm{C}_{2} \mathrm{HS}$ plus dAIH $(p<$ $0.001)$ increased phospho-TrkB immunoreactivity, and the combined effect was greater than dAIH $(p=0.002)$ or $\mathrm{C}_{2} \mathrm{HS}$ alone ( $p<0.001$; Fig. $4 l)$.

Thus, dAIH, $\mathrm{C}_{2} \mathrm{HS}$, and their combination increase the expression of key proteins necessary for intermittent hypoxia-induced spinal plasticity in both respiratory $(\mathrm{C} 4)$ and nonrespiratory motor nuclei (C7). Thus, we suggest that similar mechanisms of $\mathrm{dAIH}$ induced functional improvement of respiratory and nonrespiratory motor function.

\section{dAIH does not cause \\ hippocampal injury}

There were no dAIH-induced differences in the size, shape, density, or labeling intensity of GFAP-positive (Fig. $5 a-c$ ) or OX-42positive (Fig. $5 d-f$ ) cells. Furthermore, the number of GFAP-immunoreactive (normoxia, $284 \pm 21$; dAIH, $298 \pm 9 ; p=0.56$, $n=4$ ) and OX-42 immunoreactive (normoxia, $189 \pm 4$; dAIH, $177 \pm 10 ; p=0.29$, $n=4)$ cells were unaffected by dAIH. Neither TUNEL nor Nissl staining revealed evidence of apoptotic cell death in the hippocampal CA2 subfield (Fig. $5 g-j$ ). Collectively, our data demonstrate that $\mathrm{dAIH}$ elicits functional and neurochemical plasticity without nonspecific hippocampal pathology.

\section{Discussion}

Here we demonstrate that dAIH improves both forelimb and respiratory motor function in rodent models of chronic cervical spinal injury. Because dAIH elicits similar neurochemical plasticity in respiratory and nonrespiratory motor nuclei, common mechanisms may elicit functional consequences that differ based on the specific muscles innervated.

Although it has been known for some time that intermittent hypoxia elicits plasticity in respiratory motor function (Fuller et al., 2003; Baker-Herman et al., 2004; Golder and Mitchell, 2005), it was only recently reported that a single presentation of AIH increases ankle strength (plantar flexion torque) in humans with motor incomplete chronic spinal injuries (Trumbower et al., 2012), suggesting that both motor 
functions are affected by AIH in a similar way. The present manuscript provides the first demonstration of the following: (1) modest protocols of repetitive intermittent hypoxia elicit functional recovery in unanesthetized rats after chronic spinal injury; (2) intermittent hypoxia induces similar functional and neurochemical plasticity in respiratory and somatic motor systems; and (3) repetitive AIH elicits plasticity without comorbidities characteristic of more severe protocols of $\mathrm{CIH}$ (e.g., hypertension and hippocampal cell death). Repetitive AIH, particularly when combined with skilled motor training, has considerable promise as a therapeutic approach in the treatment of chronic spinal injury, a condition for which there are no effective treatments and no known cures.

\section{Intermittent hypoxia stimulates functional recovery of breathing capacity}

Collectively, our results confirm that dAIH elicits spinal respiratory plasticity (Wilkerson and Mitchell, 2009) and extends these reports by demonstrating that $\mathrm{dAIH}$ restores breathing capacity in spinally injured rats. This functional restoration is attributable, at least in part, to strengthening crossed-spinal synaptic pathways to phrenic motor neurons (Goshgarian, 1981, 2003; Ling et al., 1994; Golder et al., 2001a,b; Nantwi and Goshgarian, 2001). Similar functional recovery in unanesthetized, spontaneously breathing rats has not been demonstrated previously. Specific evidence for spinal plasticity (vs other CNS sites) includes the following: (1) increased spontaneous respiratory phrenic nerve activity below the hemisection (Fig. 2); (2) enhanced short-latency inputs from the contralateral ventrolateral funiculus to phrenic motor neurons (Fig. 2); and (3) increased BDNF, TrkB, and phospho-TrkB expression in phrenic motor nuclei (Fig. 4). Even a single AIH presentation was sufficient to restore baseline phrenic nerve activity in rats 1 month after cervical spinal injury, although the duration of these effects is quite limited. In addition, AIH-induced functional recovery is not observed 2 weeks after injury (Golder and Mitchell, 2005) without repetitive intermittent hypoxia exposures (Figs. 1, 2). Although dAIH induces spinal respiratory plasticity (Figs. 2, 4), additional effects at other sites are possible. For example, severe $\mathrm{CIH}$ protocols elicit plasticity at multiple sites of respiratory control (Mitchell and Johnson, 2003), including peripheral chemoreceptors (Rey et al., 2004; Pawar et al., 2008; Peng et al., 2001) and brainstem respiratory neurons (Ling et al., 2001; Kline et al., 2007).

\section{dAIH elicits prolonged recovery of skilled forelimb function}

Beginning 4 weeks after injury, individual AIH sessions were followed ( $1 \mathrm{~h}$ later) by assessment of skilled forelimb function (horizontal ladder walking). Thus, it is somewhat difficult to discriminate the effects of AIH per se versus paired training (AIH plus ladder walking). Similarly, it is difficult to differentiate the impact of dAIH versus repeatedly stimulating breathing during hypoxia on subsequently increased breathing capacity. We speculate that $\mathrm{dAIH}$ is more effective at improving motor function or the persistence of functional recovery if used as a preconditioning treatment for task-specific rehabilitation.

Although we cannot make specific conclusions concerning cellular mechanisms giving rise to improved forelimb function during dAIH treatment, a mechanism similar to that known to underlie respiratory motor plasticity (Mahamed and Mitchell, 2007; Vinit et al., 2009; Dale-Nagle et al., 2010) is likely to underlie plasticity and functional recovery in the rodent forelimb. Spinal serotonin receptor activation is necessary and sufficient for intermittent hypoxia-induced phrenic motor facilitation (Baker-Herman and Mitchell, 2002; Lovett-Barr et al., 2006; MacFarlane and Mitchell, 2009). Future studies are needed to address the hypothesis that AIH triggers episodic serotonin release near forelimb motor neurons, activating serotonin receptors and triggering a cellular cascade, leading to increased BDNF synthesis, TrkB activation, and downstream events that functionally enhance synaptic inputs to relevant motor neurons. Similar neurochemical changes (BDNF, TrkB, and phospho-TrkB) in C4 and C7 motor nuclei (Fig. 4) are consistent with this hypothesis.

Although more severe protocols of intermittent hypoxia elicit spinal plasticity and improve respiratory motor output below a spinal injury (Fuller et al., 2003), such protocols may be limited as a therapeutic tool because they also elicit multiple forms of pathology, including hypertension (Prabhakar and Kumar, 2004; Sajkov and McEvoy, 2009) and cognitive deficits attributable to brain inflammation and hippocampal cell death (Gozal et al., 2001; Li et al., 2003; Xu et al., 2004; Prabhakar et al., 2005; Zieliński, 2005; Gozal and Kheirandish-Gozal, 2007). The more moderate dAIH does not elicit hypertension (Wilkerson and Mitchell, 2009). Furthermore, we demonstrate here that dAIH does not elicit reactive gliosis based on hippocampal expression of proteins unique to astrocytes (GFAP) or microglia (cd11b; OX-42 antibody). Using TUNEL and Nissl staining, we also provide evidence that $\mathrm{dAIH}$ is not sufficiently strong to induce hippocampal cell death (Fig. 5) and presumably cognitive deficits.

\section{Significance}

The evidence presented here suggests that repetitive exposure to $\mathrm{AIH}$ is a viable therapeutic approach to treat spinal injury. Indeed, in persons with incomplete, chronic SCI (American Spinal Injury Association impairment scale $\mathrm{C}$ or $\mathrm{D}$; average of 15 years after injury), even a single presentation of AIH increases plantar flexion torque by $82 \%$, an effect that lasts $\geq 4 \mathrm{~h}$ (Trumbower et al., 2012). However, certain issues must be resolved before translation to clinical application can be realized. Targeted investigations concerning the potential of repetitive $\mathrm{AIH}$ to facilitate restoration of respiratory and limb function in safe and meaningful ways must be investigated. To this end, it will be useful to determine optimal protocols of intermittent hypoxia for motor improvement, including the severity, the pattern, and the number of hypoxic episodes presented to the patient (Vinit et al., 2009). The pattern and number of episodes are key, because respiratory plasticity is highly pattern sensitive (Mitchell et al., 2001); continuous hypoxia does not elicit similar plasticity (Baker and Mitchell, 2000; Olson et al., 2001). A balance must be achieved between maximal improvement in motor function and minimization of adverse consequences, such as neuronal cell death and learning deficits (Gozal et al., 2001; Gozal and Kheirandish-Gozal, 2007) or hypertension and autonomic dysreflexia (Prabhakar and Kumar, 2004; Sajkov and McEvoy, 2009). It is also important to understand conditions that may undermine the therapeutic efficacy of intermittent hypoxia. For example, systemic inflammation (a prominent occurrence after SCI) undermines hippocampal, spinal motor, and respiratory plasticity (Di Filippo et al., 2008; Hook et al., 2008, Vinit et al., 2011). Ultimately, the success (or failure) of intermittent hypoxia as a 
treatment to restore motor function in persons with SCI will depend on multiple factors.

\section{References}

Baker TL, Mitchell GS (2000) Episodic but not continuous hypoxia elicits long-term facilitation of phrenic motor output in rats. J Physiol 529:215-219.

Baker-Herman TL, Mitchell GS (2002) Phrenic long-term facilitation requires spinal serotonin receptor activation and protein synthesis. J Neurosci 22:6239-6246.

Baker-Herman TL, Fuller DD, Bavis RW, Zabka AG, Golder FJ, Doperalski NJ, Johnson RA, Watters JJ, Mitchell GS (2004) BDNF is necessary and sufficient for spinal respiratory plasticity following intermittent hypoxia. Nat Neurosci 7:48-55.

Blight AR (2004) Just one word: plasticity. Nat Neurosci 7:206-208.

Bocchiaro CM, Feldman JL (2004) Synaptic activity-independent persistent plasticity in endogenously active mammalian motoneurons. Proc Natl Acad Sci U S A 101:4292-4295.

Boulenguez P, Vinay L (2009) Strategies to restore motor functions after spinal cord injury. Curr Opin Neurobiol 19:587-600.

Boulenguez P, Gestreau C, Vinit S, Stamegna JC, Kastner A, Gauthier P (2007) Specific and artifactual labeling in the rat spinal cord and medulla after injection of monosynaptic retrograde tracers into the diaphragm. Neurosci Lett 417:206-211.

Cafferty WB, McGee AW, Strittmatter SM (2008) Axonal growth therapeutics: regeneration or sprouting or plasticity? Trends Neurosci 31:215-220.

Dale-Nagle EA, Hoffman MS, MacFarlane PM, Mitchell GS (2010) Multiple pathways to long-lasting phrenic motor facilitation. Adv Exp Med Biol 669:225-230.

Dale-Nagle EA, Satriotomo I, Mitchell GS (2011) Spinal vascular endothelial growth factor induces pireni motor facilitation via extracellular signalregulated kinase and Akt signaling. J Neurosci 31:7682-7690.

Di Filippo M, Sarchielli P, Picconi B, Calabresi P (2008) Neuroinflammation and synaptic plasticity: theoretical basis for a novel, immunecentered, therapeutic approach to neurological disorders. Trends Pharm Sci 29:402-412.

Frankel HL, Coll JR, Charlifue SW, Whiteneck GG, Gardner BP, Jamous MA, Krishnan KR, Nuseibeh I, Savic G, Sett P (1998) Long-term survival in spinal cord injury: a fifty year investigation. Spinal Cord 36:266-274.

Fuller DD, Bach KB, Baker TL, Kinkead R, Mitchell GS (2000) Long term facilitation of phrenic motor output. Respir Physiol 121:135-146.

Fuller DD, Johnson SM, Olson EB Jr, Mitchell GS (2003) Synaptic pathways to phrenic motoneurons are enhanced by chronic intermittent hypoxia after cervical spinal cord injury. J Neurosci 23:2993-3000.

Fuller DD, Golder FJ, Olson EB Jr, Mitchell GS (2006) Recovery of phrenic activity and ventilation after cervical spinal hemisection in rats. J Appl Physiol 100:800-806.

Golder FJ, Mitchell GS (2005) Spinal synaptic enhancement with acute intermittent hypoxia improves respiratory function after chronic cervical spinal cord injury. J Neurosci 25:2925-2932.

Golder FJ, Reier PJ, Davenport PW, Bolser DC (2001a) Cervical spinal cord injury alters the pattern of breathing in anesthetized rats. J Appl Physiol 91:2451-2458.

Golder FJ, Reier PJ, Bolser DC (2001b) Altered respiratory motor drive after spinal cord injury: supraspinal and bilateral effects of a unilateral lesion. J Neurosci 21:8680-8689.

Golder FJ, Ranganathan L, Satriotomo I, Hoffman M, Lovett-Barr MR, Watters JJ, Baker-Herman TL, Mitchell GS (2008) Spinal adenosine A2a receptor activation elicits long-lasting phrenic motor facilitation. J Neurosci 28:2033-2042.

Goshgarian HG (1981) The role of cervical afferent nerve fiber inhibition of the crossed phrenic phenomenon. Exp Neurol 72:211-225.

Goshgarian HG (2003) Plasticity in respiratory motor control - Invited review: the crossed phrenic phenomenon: a model for plasticity in the respiratory pathways following spinal cord injury. J Appl Physiol 94:795-810.

Gozal D, Kheirandish-Gozal L (2007) Neurocognitive and behavioral morbidity in children with sleep disorders. Curr Opin Pulm Med 13:505-509.

Gozal D, Daniel JM, Dohanich GP (2001) Behavioral and anatomical correlates of chronic episodic hypoxia during sleep in the rat. J Neurosci 21:2442-2450.
Hook MA, Huie JR, Grau JW (2008) Peripheral inflammation undermines the plasticity of the isolated spinal cord. Behav Neurosci 122:233-249.

Kaegi S, Schwab ME, Dietz V, Fouad K (2002) Electromyographic activity associated with spontaneous functional recovery after spinal cord injury in rats. Eur J Neurosci 16:249-258.

Kanagal SG, Muir GD (2009) Task-dependent compensation after pyramidal tract and dorsolateral spinal lesions in rats. Exp Neurol 216:193-206.

Kline DD, Ramirez-Navarro A, Kunze DL (2007) Adaptive depression in synaptic transmission in the nucleus of the solitary tract after in vivo chronic intermittent hypoxia: evidence for homeostatic plasticity. J Neurosci 27:4663-4673.

Li RC, Row BW, Gozal E, Kheirandish L, Fan Q, Brittian KR, Guo SZ, Sachleben LR Jr, Gozal D (2003) Cyclooxygenase 2 and intermittent hypoxia-induced spatial deficits in the rat. Am J Respir Crit Care Med 168:469-475.

Ling L, Bach KB, Mitchell GS (1994) Serotonin reveals ineffective spinal pathways to contralateral phrenic motoneurons in spinally hemisected rats. Exp Brain Res 101:35-43.

Ling L, Fuller DD, Bach KB, Kinkead R, Olson EB Jr, Mitchell GS (2001) Chronic intermittent hypoxia elicits serotonin-dependent plasticity in the central neural control of breathing. J Neurosci 21:5381-5388.

Lovett-Barr MR, Mitchell GS, Satriotomo I, Johnson SM (2006) Serotonininduced in vitro long-term facilitation exhibits differential pattern sensitivity in cervical and thoracic inspiratory motor output. Neuroscience 142:885-892.

MacFarlane PM, Mitchell GS (2009) Episodic spinal serotonin receptor activation elicits long-lasting phrenic motor facilitation by an $\mathrm{NADPH}$ oxidase-dependent mechanism. J Physiol 587:5469-5481.

MacFarlane PM, Wilkerson JE, Lovett-Barr MR, Mitchell GS (2008) Reactive oxygen species and respiratory plasticity following intermittent hypoxia. Respir Physiol Neurobiol 164:263-271.

Mahamed S, Mitchell GS (2007) Is there a link between intermittent hypoxia-induced respiratory plasticity and obstructive sleep apnoea? Exp Physiol 92:27-37.

Mantilla CB, Zhan WZ, Sieck GC (2009) Retrograde labeling of phrenic motoneurons by intrapleural injection. J Neurosci Methods 182:244-249.

Mitchell GS, Johnson SM (2003) Neuroplasticity in respiratory motor control. J Appl Physiol 94:358-374.

Mitchell GS, Baker TL, Nanda SA, Fuller DD, Zabka AG, Hodgeman BA, Bavis RW, Mack KJ, Olson EB Jr (2001) Invited review: intermittent hypoxia and respiratory plasticity. J Appl Physiol 90:2466-2475.

Muir GD, Webb AA, Kanagal S, Taylor L (2007) Dorsolateral cervical spinal injury differentially affects forelimb and hindlimb action in rats. Eur J Neurosci 25:1501-1510.

Nantwi KD, Goshgarian HG (2001) Alkylxanthine-induced recovery of respiratory function following cervical spinal cord injury in adult rats. Exp Neurol 168:123-134.

Olson EB Jr, Bohne CJ, Dwinell MR, Podolsky A, Vidruk EH, Fuller DD, Powell FL, Mitchel GS (2001) Ventilatory long-term facilitation in unanesthetized rats. J Appl Physiol 91:709-716.

Pawar A, Peng YJ, Jacono FJ, Prabhakar NR (2008) Comparative analysis of neonatal and adult rat carotid body responses to chronic intermittent hypoxia. J Appl Physiol 104:1287-1294.

Paxinos G, Watson C (1997) The rat brain in stereotaxic coordinates. San Diego: Academic

Peng Y, Kline DD, Dick TE, Prabhakar NR (2001) Chronic intermittent hypoxia enhances carotid body chemoreceptor response to low oxygen. Adv Exp Med Biol 499:33-38.

Poulton NP, Muir GD (2005) Treadmill training ameliorates dopamine loss but not behavioral deficits in hemi-parkinsonian rats. Exp Neurol 193:181-197.

Prabhakar NR, Kumar GK (2004) Oxidative stress in the systemic and cellular responses to intermittent hypoxia. Biol Chem 385:217-221.

Prabhakar NR, Peng YJ, Jacono FJ, Kumar GK, Dick TE (2005) Cardiovascular alterations by chronic intermittent hypoxia: importance of carotid body chemoreflexes. Clin Exp Pharmacol Physiol 32:447-449.

Raineteau O, Schwab ME (2001) Plasticity of motor systems after incomplete spinal cord injury. Nat Rev Neurosci 2:263-273. 
Ramer MS, Harper GP, Bradbury EJ (2000) Progress in spinal cord research: a refined strategy for the international spinal research trust. Spinal Cord 38:449-472.

Rey S, Del Rio R, Alcayaga J, Iturriaga R (2004) Chronic intermittent hypoxia enhances cat chemosensory and ventilatory responses to hypoxia. J Physiol 560:577-586.

Sajkov D, McEvoy RD (2009) Obstructive sleep apnea and pulmonary hypertension. Prog Cardiovasc Dis 51:363-370.

Trumbower RD, Jayaraman A, Mitchell GS, Rymer WZ (2012) Exposure to acute intermittent hypoxia augments somatic motor function in humans with incomplete spinal cord injury. Neurorehabil Neural Repair 26:163-172.

Vinit S, Lovett-Barr MR, Mitchell GS (2009) Intermittent hypoxia induces functional recovery following cervical spinal injury. Respir Physiol Neurobiol 169:210-217.

Vinit S, Windelborn JA, Mitchell GS (2011) Lipopolysaccharide attenuates phrenic long-term facilitation following acute intermittent hypoxia. Respir Physiol Neurobiol 176:130-135.

Webb AA, Muir GD (2003) Unilateral dorsal column and rubrospinal tract injuries affect overground locomotion in the unrestrained rat. Eur J Neurosci 18:412-422.

Wilkerson JE, Mitchell GS (2009) Preconditioning with daily acute intermittent hypoxia augments spinal BDNF levels, ERK phosphorylation and respiratory long-term facilitation in Brown Norway rats. Exp Neurol 217:116-123.

Xu W, Chi L, Row BW, Xu R, Ke Y, Xu B, Luo C, Kheirandish L, Gozal D, Liu $R$ (2004) Increased oxidative stress is associated with chronic intermittent hypoxia-mediated brain cortical neuronal cell apoptosis in a mouse model of sleep apnea. Neuroscience 12:313-323.

Zieliński J (2005) Effects of intermittent hypoxia on pulmonary haemodynamics. Pneumonol Alergol Pol 73:86-93. 